\title{
Ultrafast rerouting of light via slow modes in a nanophotonic directional coupler
}

\author{
T. Kampfrath, ${ }^{1, a)}$ D. M. Beggs, ${ }^{2}$ T. P. White, ${ }^{2}$ M. Burresi, ${ }^{1}$ D. van Oosten, ${ }^{1}$ T. F. Krauss, ${ }^{2}$ \\ and L. Kuipers ${ }^{1}$ \\ ${ }_{1}^{1}$ FOM Institute AMOLF, Science Park 113, 1098 XG Amsterdam, The Netherlands \\ ${ }^{2}$ School of Physics and Astronomy, University of St. Andrews, St. Andrews, Fife KY16 9SS, United Kingdom
}

(Received 11 March 2009; accepted 22 May 2009; published online 19 June 2009)

\begin{abstract}
We demonstrate that two coupled photonic-crystal waveguides can route two subsequently arriving light pulses to different output ports even though the pulses are only 3 ps apart. This rerouting of light is due to an ultrafast shift in the transmittance spectrum triggered by the generation of electrons and holes in the Si base material by a femtosecond laser pulse. The use of slow-light modes allows for a coupler length of only $5.2 \mu \mathrm{m}$. Since these modes are not directly involved, the $3 \mathrm{ps}$ dead time is solely determined by the duration of the input pulse rather than its transit time through the device. (C) 2009 American Institute of Physics. [DOI: 10.1063/1.3153989]
\end{abstract}

Future data networks operating at speeds of terabits per second will be based on photons as information carriers rather than electrons. Pioneering work on the way to this technology has demonstrated nanophotonic devices that can switch their light transmission on or off on a picosecond time scale, for instance, based on ring resonators ${ }^{1-3}$ or MachZehnder interferometers. ${ }^{4}$ Recently, an alternative design has been proposed, which relies on mode interference in two side-coupled waveguides to selectively route light to different output ports. ${ }^{5}$ Due to the use of slow-light modes in photonic-crystal waveguides, the practical realization ${ }^{6,7}$ of such a nanophotonic directional coupler (DC) features a total footprint as small as $10 \mu \mathrm{m} \times 10 \mu \mathrm{m}$. However, its capability to reroute light has so far only been demonstrated on a microsecond time scale by modulating the device temperature. $^{7}$

In this letter, we employ femtosecond laser pulses to investigate how fast the DC can be operated. We show that the device can route two subsequently arriving light pulses to different output ports although they are only 3 ps apart. Since the slow-light modes of the device are only indirectly involved, the device speed is not limited by the transit time but solely by the duration of the input pulse itself and, thus, the optical bandwidth of the coupler.

Our DC is schematically shown in Fig. 1(a). It consists of two parallel waveguides embedded in a $\mathrm{Si}$ photoniccrystal membrane. As in a conventional DC, side-coupling of these waveguides leads to the formation of two Bloch supermodes with frequency $\omega$ and wavevector $k$. The electric field of the first mode is symmetric with respect to the symmetry plane between the two waveguides, whereas the second mode is antisymmetric. The dispersion relation $\omega(k)$ of both modes is sketched in Fig. 1(b). When a probe pulse with center frequency $\omega$ is fed into input port IN1, a superposition of the symmetric and antisymmetric mode is created. Since the modes exhibit a wavevector difference $\Delta k$, the resulting light field will spatially beat between the two waveguides. ${ }^{5,8}$ In particular, after a propagation length of $\pi / \Delta k$, the light intensity is mainly localized in the second waveguide. The photonic-crystal environment allows for a tailored dispersion

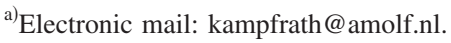

such as in Fig. 1(b). The slow-light modes with flat slope $v_{\mathrm{g}}=\partial \omega / \partial k$ lead to a large increase in $\Delta k$ when the frequency decreases from $\omega_{1}$ to $\omega_{2}$. In our device, light at frequency $\omega_{2}$ is transferred to the second waveguide after only $5.2 \mu \mathrm{m}$ of propagation, whereas light at the slightly higher frequency $\omega_{1}$ is still in the first waveguide. Therefore, light of frequency $\omega_{1}$ or $\omega_{2}$ will exit the DC at output port OUT1 or OUT2, respectively. In order to reroute light of frequency $\omega_{1}$ to output port OUT2, the dispersion relation has to be shifted up by some frequency $\omega_{1}-\omega_{2}$. In our experiment, this shift is realized by changing the refractive index of the Si base material via the absorption of an ultrashort pump pulse.
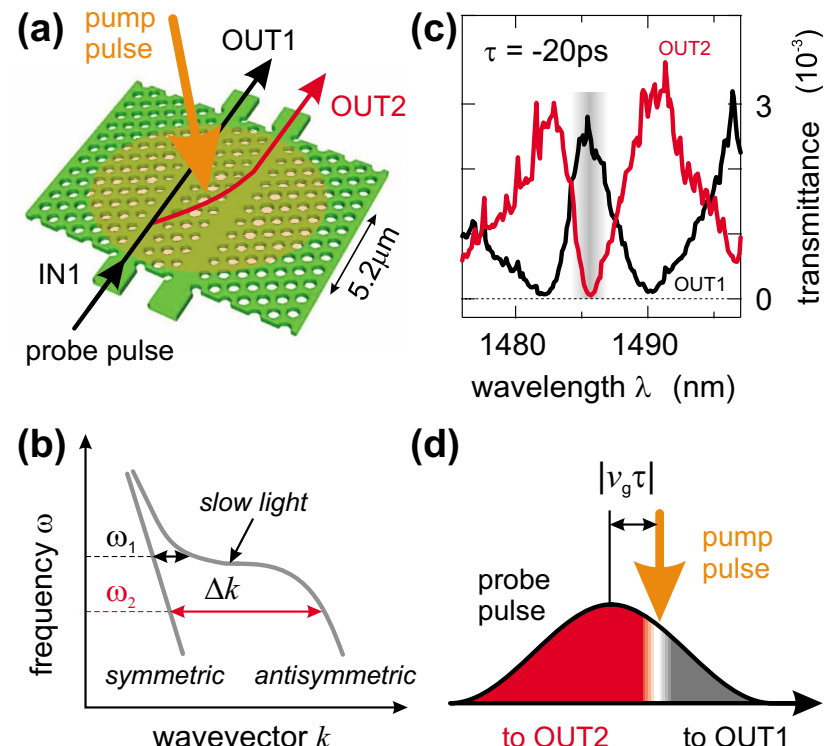

(d)

FIG. 1. (Color online) (a) Schematic of the ultrafast rerouter. A probing light pulse enters a photonic-crystal based DC at input port IN1 and is coupled out at output port OUT1. Illumination of the device by a pump pulse reroutes subsequent probe pulses to output port OUT2. (b) Dispersion relation of the symmetric and antisymmetric Bloch supermode of the two sidecoupled waveguides. (c) Transmittance spectrum of the DC at $20 \mathrm{ps}$ before arrival of the pump pulse. The shaded area indicates the spectral range of the probe pulse used in the switching experiments. (d) Illustration of the rerouting process. Probe light which leaves the DC before excitation by the pump pulse exits at OUT1, and probe light arriving at the DC after excitation exits at OUT2. 
Details on the fabrication and characterization of the DC can be found in Refs. 6 and 7. In order to increase mechanical stability and to provide an appropriate heat sink, the 220 $\mathrm{nm}$ thin $\mathrm{Si}$ membrane is embedded in $\mathrm{SiO}_{2} .{ }^{9}$ The DC input and output ports are connected to Si ridge waveguides, and optimum coupling of light into and out of the $5.2 \mu \mathrm{m}$ long DC region is enabled by two $2.4 \mu \mathrm{m}$ long interface regions. The Si membrane is illuminated from above, as sketched in Fig. 1(a), by pump pulses with a duration of 100 fs full width at half maximum of the intensity (FWHMI), a center wavelength of $800 \mathrm{~nm}$, and a repetition rate of $80 \mathrm{MHz}$ from a Ti:sapphire laser oscillator. The excited $\mathrm{Si}$ area has a diameter of about $20 \mu \mathrm{m}$ FWHMI such that the absorption of each pump pulse leads to the generation of a homogeneous electron-hole plasma in the Si parts of the DC. We estimate that about $1 \%$ of the incident pump power is absorbed in the $\mathrm{Si}$ membrane. The instantaneous device transmittance is probed by laser pulses with a variable bandwidth from 1 to $20 \mathrm{~nm}$ FWHMI and a center wavelength of $1486 \mathrm{~nm}$, which are derived from the same laser system using an optical parametric oscillator and a grating-based reflective pulse shaper. ${ }^{10}$ These probe pulses are delayed with respect to the pump pulses by an adjustable delay and coupled into input port IN1. The transmitted probe light is picked up either at output port OUT1 or OUT2 by a lensed fiber and fed into an optical spectrum analyzer. In this way, we can record the transmitted probe spectrum as a function of the delay $\tau$ between pump and probe pulse. Here, $\tau=0$ means that pump and probe pulse arrive at the same time at the center of the DC.

Figure 1(c) shows the transmittance of the DC as a function of wavelength $\lambda=2 \pi c / \omega$ at $\tau=-20 \mathrm{ps}$, that is, for an unexcited sample, since the probe pulse arrives 20 ps before the pump pulse. Note the spectral oscillations that exhibit a complementary behavior for output ports OUT1 and OUT2. For example, in the shaded region around $\lambda=1486 \mathrm{~nm}$ of Fig. 1(c), the transmittance from IN1 to OUT1 is maximum, while it is nearly zero from IN1 to OUT2. We now use a 1.2 $\mathrm{nm}$ FWHMI wide probe pulse in order to investigate the dynamics of the transmitted light at this specific wavelength. Assuming a bandwidth-limited Gaussian pulse, this width corresponds to a pulse duration of 2.8 ps FWHMI.

Figure 2 shows the key results of this experiment. The colorscale plots of Figs. 2(a) and 2(b) display the probe intensity spectrum detected at OUT1 and OUT2, respectively, as a function of wavelength $\lambda$ and the pump-probe delay $\tau$. For delays $\tau<-2$ ps, the probe pulse sees an unexcited DC and thus arrives at OUT1, whereas no intensity arrives at OUT2. The situation reverses for delays $\tau>2$ ps at which the IN1-to-OUT1 path is closed but the IN1-to-OUT2 path opened. Figure 2(c) displays the total probe power arriving at OUT1 and OUT2 as a function of the pump-probe delay. Note that the two signals change by more than $80 \%$ within less than 3 ps. In other words, our DC can route subsequently arriving light pulses to different output ports even when the pulses are separated by less than 3 ps.

What determines the $3 \mathrm{ps}$ "dead time" of the rerouting process? Upon arrival of the pump pulse, the refractive index of the Si jumps to a new value within the 100 fs duration of the pump pulse, ${ }^{11}$ and so do the instantaneous transmission properties of the DC. Therefore, as illustrated by Fig. 1(d), the leading part of the probe pulse, which has already left the DC before arrival of the pump pulse, is coupled out at
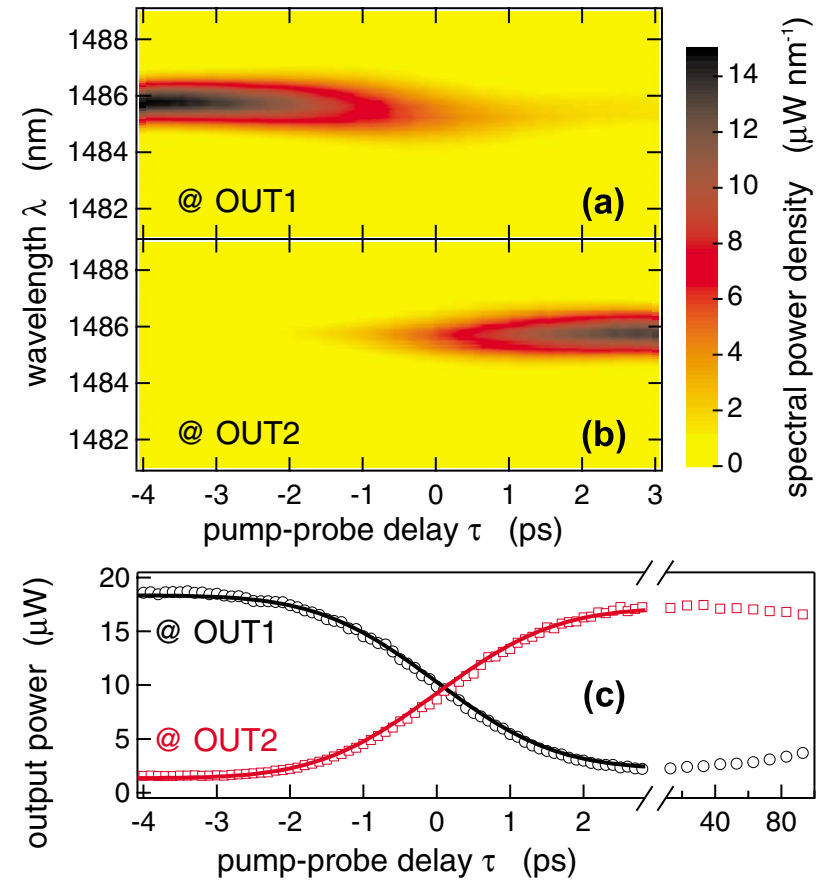

FIG. 2. (Color online) Experimental data demonstrating the ultrafast rerouting of light for an absorbed pump energy of about 6 pJ. (a) Spectra of the probe pulses arriving at output port OUT1 as a function of the pump-probe delay $\tau$. (b) Same as (a) for output port OUT2. (c) Measured total probe power arriving at OUT1 and OUT2 as a function of $\tau$ (symbols). Thick solid lines are calculations based on Eq. (1).

OUT1. Similarly, the trailing part of the probe pulse which has not yet entered the DC will be directed to OUT2. The remaining part of the probe pulse that is just inside the DC when being excited by the pump pulse can be neglected. This is justified since the transit time $L / v_{\mathrm{g}}=0.1 \mathrm{ps}$ of the probe pulse through the DC is much smaller than the 2.8 ps duration of the probe pulse. Here, $L=5.2 \mu \mathrm{m}$ is the DC length and $v_{\mathrm{g}}=0.15 c$ the group velocity, as estimated from the band structure of the DC. ${ }^{6}$ As a consequence, the probe power arriving at OUT2 is proportional to the red part of the pulse area in Fig. 1(d), and we have

$$
\text { probe power at OUT2 } \propto \int_{-\infty}^{\tau} \mathrm{d} t I(t) .
$$

Here, $I(t)$ is the instantaneous intensity of the incident Gaussian probe pulse with a duration of 2.8 ps FWHMI centered at time $t=0$. The probe power at OUT1 is obtained by a similar calculation. Figure 2(c) shows excellent agreement with the experimental data. Therefore, the dead time of the ultrafast rerouter is solely determined by the duration of the probe pulse. This finding seems counterintuitive as our DC is based on slow light. However, as seen in Fig. 1(b), the slowlight modes are not populated by the probe pulse and participate only indirectly by inducing large wavevector changes for small frequency shifts. Note that the DC literally "cuts" the incident probe pulse in two shorter pulses in the vicinity of $\tau=0$. This notion is consistent with the broader measured transmission spectra at these small pump-probe delays in Figs. 2(a) and 2(b).

Figure 2(c) also shows the dynamics of the DC transmittance on longer time scales. The data suggest that the DC relaxes into its initial state on a time scale of about $0.4 \mathrm{~ns}$. 


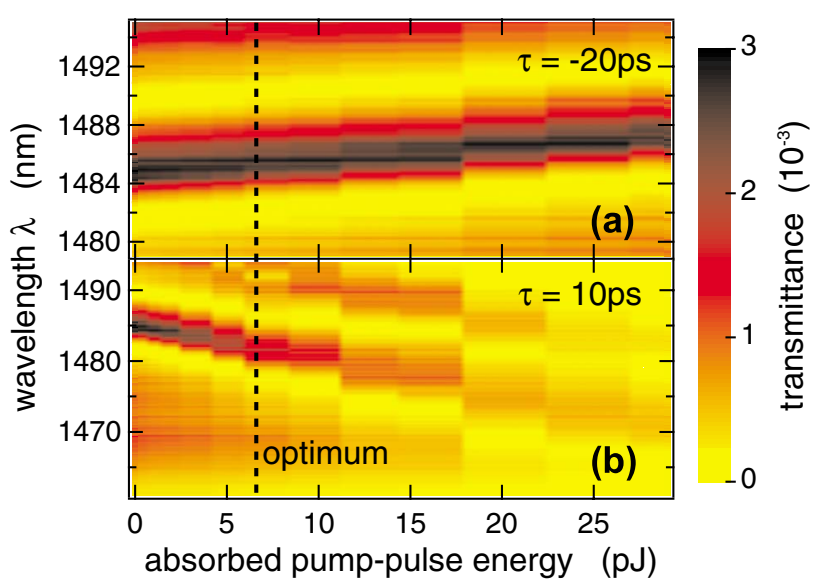

FIG. 3. (Color online) Spectral transmittance from IN1 to OUT1 as a function of the absorbed pump-pulse energy at (a) $\tau=-20$ ps and (b) $\tau=10$ ps. Note the different ordinate scalings.

This decay is governed by the lifetime of the pumpgenerated free electrons and holes. ${ }^{11}$

In order to understand the switch mechanism of the DC in more detail, we have also measured the DC transmittance with broadband probe pulses at various pump-pulse energies. Figure 3(a) displays the transmittance for a pump-probe delay of $\tau=-20 \mathrm{ps}$, that is, long before arrival of the pump pulse. The rigid redshift in the transmittance spectra with increasing pump energy arises, since the cumulative absorption of pump pulses with a repetition rate of $80 \mathrm{MHz}$ leads to a stationary increase $\Delta T$ in the DC temperature. The resulting refractive-index change $\Delta n=\kappa \Delta T$ of the Si exceeds that of the $\mathrm{SiO}_{2}$ by far since the thermo-optic coefficient $\kappa=1.8$ $\times 10^{-4} \mathrm{~K}^{-1}$ of $\mathrm{Si}$ is more than one order of magnitude larger. ${ }^{12,13}$ We will therefore only consider the effects of the refractive-index change $\Delta n$ of the $\mathrm{Si}$ in the following. As can be shown by perturbation theory, ${ }^{14} \Delta n$ induces a shift in the frequency $c / \lambda_{r}$ of each DC eigenmode $r$, which is determined by

$$
\frac{\Delta \lambda_{r}}{\lambda_{r}}=p_{r} \frac{\Delta n}{n} .
$$

Here, $0 \leq p_{r} \leq 1$ is the fraction of the mode energy that is concentrated in the Si parts of the DC. Provided the shifted modes couple as efficiently to the connected ridge waveguide as the unperturbed modes, the entire transmission spectrum of the DC undergoes a rigid wavelength shift $\Delta \lambda$ of the order of $\lambda \Delta n / n$. Since $\kappa$ is positive in $\mathrm{Si}$, an increasing temperature induces a redshift in the transmission spectrum, in agreement with the experimental data in Fig. 3(a). For the rerouting results of Fig. 2, the observed redshift in $\Delta \lambda$ $=0.35 \mathrm{~nm}$ implies that the DC temperature has increased by roughly $4 \mathrm{~K}$.

Finally, Fig. 3(b) shows transmittance spectra for a positive pump-probe delay of $\tau=10 \mathrm{ps}$, that is, long after arrival of the pump pulse. In contrast to Fig. 3(a), we now observe a blueshift in the spectra and, in addition, a reduction and broadening in the transmittance peaks with increasing pump energy. At this delay, the pump-induced change $\Delta n$ in the refractive index is dominated by the Drude-type conductivity of the electron-hole plasma, ${ }^{11}$ which implies a negative $\operatorname{Re} \Delta n$ and thus a blueshift in the DC eigenmodes. However, the existence of free electrons and holes in the $\mathrm{Si}$ also opens new channels for the absorption of the probe light, ${ }^{15}$ and one has a positive $\operatorname{Im} \Delta n$. We attribute the observed reduction and broadening in the transmittance peaks to such freecarrier absorption. Alternative mechanisms for decreased transmittance are an enhanced reflectivity of the DC input or an increased light scattering out of the device plane. We can rule these mechanisms out since they should then be also operative for the redshifted data in Fig. 3(a), and lead to a similar peak reduction and broadening, which is not observed. For our DC, the optimum shift is $\Delta \lambda=-5 \mathrm{~nm}$ at which the additional losses due to free-carrier absorption are still acceptable. We summarize that the switching action of the DC can be understood as an ultrafast blueshift in the unperturbed transmittance spectrum shown in Fig. 1(c).

In conclusion, we have shown that we can reroute light from one output port of a $5.2 \mu \mathrm{m}$ short DC to another output port as fast as 3 ps. In contrast to alternative designs, ${ }^{2-4}$ the device speed is not limited by the transit time but solely by the duration of the input pulse itself. Therefore, a faster rerouter can only be realized by increasing the usable input bandwidth of the device. Our results also suggest the use of the DC as a nanophotonic pulse cutter.

We acknowledge funding through the EU FP6-FET "SPLASH" project. This work is also part of the research program of FOM, which is financially supported by the NWO.

${ }^{1}$ Y. Vlasov, W. M. J. Green, and F. Xia, Nat. Photonics 2, 242 (2008).

${ }^{2}$ V. R. Almeida, C. A. Barrios, R. R. Panepucci, and M. Lipson, Nature (London) 431, 1081 (2004).

${ }^{3}$ M. Waldow, T. Plötzing, M. Gottheil, M. Först, J. Bolten, T. Wahlbrink, and H. Kurz, Opt. Express 16, 7693 (2008).

${ }^{4}$ H. Nakamura, Y. Sugimoto, K. Kanamoto, N. Ikeda, Y. Tanaka, Y. Nakamura, S. Ohkouchi, Y. Watanabe, K. Inoue, H. Ishikawa, and K. Asakawa, Opt. Express 12, 6606 (2004).

${ }^{5}$ N. Yamamoto, T. Ogawa, and K. Komori, Opt. Express 14, 1223 (2006).

${ }^{6}$ D. M. Beggs, T. P. White, L. O'Faolain, and T. F. Krauss, Opt. Lett. 33, 147 (2008).

${ }^{7}$ D. M. Beggs, T. P. White, L. Cairns, L. O'Faolain, and T. F. Krauss, IEEE Photonics Technol. Lett. 21, 24 (2009).

${ }^{8}$ R. J. P. Engelen, Y. Sugimoto, H. Gersen, N. Ikeda, K. Asakawa, and L. Kuipers, Nat. Phys. 3, 401 (2007).

${ }^{9}$ T. P. White, L. O'Faolain, J. Li, L. C. Andreani, and T. F. Krauss, Opt. Express 16, 17076 (2008).

${ }^{10}$ R. D. Nelson, D. E. Leaird, and A. M. Weiner, Opt. Express 11, 1763 (2003).

${ }^{11}$ A. J. Sabbah and D. M. Riffe, Phys. Rev. B 66, 165217 (2002).

${ }^{12}$ J. A. McCaulley, V. M. Donnelly, M. Vernon, and I. Taha, Phys. Rev. B 49, 7408 (1994).

${ }^{13}$ J. H. Wray and J. T. Neu, J. Opt. Soc. Am. 59, 774 (1969).

${ }^{14}$ S. G. Johnson, M. Ibanescu, M. A. Skorobogatiy, O. Weisberg, J. D Joannopoulos, and Y. Fink, Phys. Rev. E 65, 066611 (2002).

${ }^{15}$ R. von Baltz and W. Escher, Phys. Status Solidi B 51, 499 (1972). 